\title{
Actualización: Tratamiento de los pacientes con trastornos alimentarios
}

\author{
Treatment of patients with eating disorders
}

Esteban Kuten* y Augusto Granel **

\begin{abstract}
Resumen
Este artículo explica las principales herramientas para el tratamiento de la anorexia y la bulimia nerviosa y la evidencia que las respalda: 1) los criterios para la decisión del ámbito de tratamiento (estado clínico, peso, comorbilidades, riesgo suicida, motivación, introvisión o "insight" y cooperación, capacidad para manejar los pensamientos intrusivos y la compulsión a realizar ejercicio, necesidad de soporte para alimentarse, red social de soporte); 2) el rol del equipo tratante; 3) la técnica de terapia familiar de Maudsley para el tratamiento de la anorexia nerviosa -que enfatiza en la restauración del peso y la seguridad física del paciente por sobre el tratamiento de una presunta disfunción familiar y pone el foco en la consideración de la anorexia nerviosa como una enfermedad o estado, por sobre una característica estructural-; 4) la técnica cognitivo-comportamental para el tratamiento de la bulimia -que enfatiza sobre los factores y procesos que mantienen y perpetúan el problema, por sobre los que lo iniciaron; y además de un cambio en la conducta alimentaria y el aprendizaje de las situaciones gatillo que precipitan los atracones, pretende una reestructuración cognitiva-;y 5) algunas pautas generales para la indicación de farmacoterapia.

\section{Abstract}

This article explains the main evidence-based therapeutic tools for Anorexia Nervosa and Bulimia Nervosa:

1) Place of treatment decision criteria (clinical status, weight, comorbidities, suicidal risk, treatment motivation, insight capability, willingness to cooperate with treatment, capability of managing intrusive thoughts, exercise compulssion, need of feeding support, social network); 2)Rol of treatment team, 3)Maudsley's family therapy treatment technique for Anorexia Nervosa management-centred on body weight restorance and physical safety of patients instead of family disfunction, considering Anorexia Nervosa a disease or state, and not a structural feature-; 4) Cognitive-behavioral technique for Bulimia Nervosa treatment-based on managing perpetuating rather than initiation factors, feeding behavioral changes, recognization of trigger situations of binge eating and cognitive restructuration-5) general guidelines for pharmacotherapy.
\end{abstract}

Palabras claves: ttratamiento trastornos alimentarios, tratamiento bulimia, tratamiento anorexia, bulimia nerviosa, anorexia nerviosa, realimentación. Kuten E y Granel A. Tratamientos de los pacientes con transtomos alimentarios. Evid. actual. práct. ambul; 10(3): 81-84 may-jun.2007.

\section{Ámbito inicial de tratamiento}

En términos generales, la mayoría de los tratamientos pueden efectuarse en forma ambulatoria, reservándose el tratamiento intensivo en hospital de día o en internación hospitalaria ante el fracaso del tratamiento ambulatorio.

La decisión de la internación está basada en factores psiquiátricos y en aquellos derivados de la clínica general como la corrección de trastornos del medio interno, la nutrición y la realimentación ${ }^{1}$. La hospitalización no es obligatoria ni constituye el único marco posible. Es una herramienta pero no debe emplearse como amenaza. Si bien el seguimiento de los parámetros clínicos y la información proveniente de estudios complementarios resultan indispensables en ocasiones, el exceso de prescripciones, la vigilancia constante y la pre- sentación al paciente y familiares de las posibles complicaciones, pueden tener efectos contraproducentes como la acentuación del fenómeno de negación y la descompensación depresiva ${ }^{2}$. En cuanto a la hospitalización parcial y los programas de hospital de día, estos son cada vez más usados con la idea de disminuir algunas internaciones prolongadas o para casos no tan graves. Existe un delicado equilibrio que da cuenta de que el trabajo debe ser interdisciplinario y con metas globales. Por ejemplo, no tiene sentido decir en un paciente con anorexia que está progresando "psicológicamente" si no puede evidenciarse una ganancia de peso aceptable por fuera de parámetros riesgosos. Por otro lado, también es negativa una internación con mucho sesgo biomédico que no contemple los aspectos emocionales. El cuadro 1 resume las variables de ayuda para definir el nivel de cuidado para cada paciente.

Cuadro 1: información de ayuda para definir el nivel de cuidado para cada paciente'1.

\begin{tabular}{|c|c|}
\hline Status clínico & $\begin{array}{l}\text { Desde estable clínicamente, hasta la necesidad de control de laboratorio diario del medio interno, sonda nasogástrica y/ó fluidos intravenosos. } \\
\text { Son criterios de gravedad en adultos: 1) frecuencia cardíaca menor a } 40 \text { latidos por minuto, 2) tensión arterial menor a 90/60mmHg, 3) } \\
\text { glucemia menor a } 60 \mathrm{mg} / \mathrm{dL} \text {, 4) hipokalemia, 5) hipofosfatemia,6) hipomagnesemia, 7) temperatura menor a } 36 \quad \mathrm{C}, 8) \text { deshidratación. }\end{array}$ \\
\hline Riesgo suicida & Puede no haber plan, haber plan pero no intento o tener plan y haberlo intentado. \\
\hline Peso ideal & Es de gravedad cuando es menor a $85 \%$ del peso ideal con negación a alimentarse por sí mismo. \\
\hline Motivación y cooperación. & Niveles de cooperación: 1) cooperación espontánea; 2) sólo si el tratamiento es muy estructurado; 3) no coopera. \\
\hline Introvisión. & $\begin{array}{l}\text { La introvisión } 0 \text { "insight" se refiere al proceso emocional y cognitivo que se refiere al darse cuenta de una situación problemática } 0 \\
\text { conflictiva. }\end{array}$ \\
\hline $\begin{array}{l}\text { Capacidad para controlar } \\
\text { pensamientos obsesivos }\end{array}$ & Nivel de pensamientos intrusivos y repetitivos: 1) desde ninguno, 2) hasta tenerlos más de seis horas diarias. \\
\hline Comorbilidades & Según la comorbilidad (ej. depresión, ansiedad, abuso de sustancias) se optará por el nivel de cuidado, según indicación precisa y especfica. \\
\hline $\begin{array}{l}\text { Necesidad de soporte para } \\
\text { comer y/o ganar peso por } \\
\text { si mismo. }\end{array}$ & $\begin{array}{l}\text { Puede ir desde ser autosuficiente para alimentarse, hasta necesitar soportes y supervisión, por ejemplo hasta el extremo de que se precise } \\
\text { sonda nasogástrica. }\end{array}$ \\
\hline $\begin{array}{l}\text { Control del ejercicio } \\
\text { compulsivo }\end{array}$ & $\begin{array}{l}\text { Desde la realización de ejercicio en forma saludable con control de la compulsión al mismo, hasta la necesidad de contención y estructura } \\
\text { para controlar el ejercicio compulsivo. }\end{array}$ \\
\hline $\begin{array}{l}\text { Red de soporte emocional } \\
\text { del entorno }\end{array}$ & $\begin{array}{l}\text { Desde una adecuada red psicosocial de sostén; pasando por la presencia de conflictos familiares graves, falta de un soporte para el } \\
\text { tratamiento; hasta el extremo del paciente completamente aislado. }\end{array}$ \\
\hline
\end{tabular}

* Médico Especialista en Medicina Familiar y en Psiquiatría. Servicio de Medicina Familiar y Comunitaria del Hospital Italiano de Buenos Aires.

** Médico Especialista en Medicina Familiar. Servicio de Medicina Familiar y Comunitaria del Hospital Italiano de Buenos Aires. 


\section{Manejo psiquiátrico general}

El abordaje psicoterapéutico es la base del tratamiento de los trastornos alimentarios ${ }^{1}$. Siempre que resulte posible, la terapia de familia debería ser considerada en todos los casos sobre todo para niños y adolescentes que todavía viven con sus padres $^{3}$. El tratamiento familiar demostró evitar la internación, resultó en un manejo eficaz de la enfermedad (tanto en la salud física como psicológica individual) y en una mejoría familiar ${ }^{4}$. A pesar de estas consideraciones sobre la familia, no debe focalizarse sólo en la influencia patógena sino, en especial, en su función de aliado terapéutico ${ }^{5}$.

La formación de una fuerte alianza terapéutica, la coordinación del cuidado y la colaboración con otras especialidades clínicas son fundamentales para realizar un seguimiento del comportamiento y del monitoreo de las condiciones clínicas generales. Se debe promover la creación de una alianza terapéutica realizando una evaluación familiar al inicio del tratamiento, haciendo participar a la familia en el mismo y evaluando junta a ella la personalidad del paciente, los conflictos familiares que favorecen el trastorno y los conflictos en los cuales el paciente participa $^{34}$.

Los consejos y el seguimiento dietético pueden formar parte de la restauración de la normal alimentación. Debe tenerse especial precaución con las prescripciones dietéticas estrictas ya que pueden reforzar las obsesiones alimentarias y contribuir a mantener o inclusive, empeorar el problema.

Un programa de alimentación en forma de contrato abierto, con instrucciones precisas como un cuaderno alimentario son un método didáctico de gran utilidad como forma de reestructuración cognitiva dirigida a mejorar las distorsiones de la imagen del cuerpo y la relación con el alimento ${ }^{4}$.

Durante todo el tratamiento es necesario apoyar los aspectos positivos que refuercen la autoestima por sobre la interpretación de los conflictos ${ }^{3}$. La psicoterapia debe realizarse en todos los pacientes, combinada con otras modalidades terapéuticas.

El cuadro 2 resume el rol del equipo tratante en el manejo del paciente con trastornos de la alimentación.

Cuadro 2: rol del equipo tratante en el manejo del paciente con trastornos de la alimentación'.

- Establecer y mantener una alianza terapéutica

- Coordinación del cuidado con otras especialidades

- Evaluar y monitorear los síntomas y conductas relacionadas al trastorno alimentario

- Evaluar y monitorear las condiciones clínicas generales

- Participar a la familia como parte integral del tratamiento

\section{Tratamientos específicos para los pacientes con anorexia nerviosa}

Los objetivos en el tratamiento de la anorexia nerviosa (AN) incluyen la restauración del peso saludable. En las mujeres se refiere al peso en el cual la menstruación, los niveles hormonales y la ovulación se encuentran conservados; en los niños y adolescentes, el que se asocia a un crecimiento físico y desarrollo sexual normal.

Son también objetivos del tratamiento tratar las complicaciones físicas, que el paciente retome las motivaciones por modelos de alimentación sanos y abordar condiciones psiquiátricas comórbidas $^{56}$.
Psicoterapia

Si bien en pacientes adultos con AN los resultados son inconclusos, hay numerosos estudios que han demostrado la utilidad de la terapia familiar en adolescentes. La guía de práctica del Reino Unido (NICE) ha evaluado la utilización de la terapia familiar para AN como una recomendación de evidencia grado $B$, debido a que se cuenta con evidencia proveniente de ensayos clínicos bien diseñados pero no aleatorizados ${ }^{7}$.

Los primeros trabajos fueron realizados por Minuchin y colaboradores en el Child Guidance Clinic. Si bien poseían limitaciones metodológicas ya que no contaron con grupos controles y tuvieron tiempos variables de seguimiento, estos estudios resultaron pioneros.

Tomando este modelo como base, se desarrolló posteriormente el método Maudsley (por el Hospital de Londres que lleva su nombre), la modalidad de tratamiento actualmente más difundida ${ }^{8}$. Se trata de un método estructurado o manualizado de terapia familiar, que ha sido probado exitosamente en varios lugares y adaptado recientemente a pacientes internados de mayor gravedad. Basa el tratamiento en la interacción con la familia, ubicando el problema fuera de ella y trabajando sobre la toma de decisiones y el control. Se redefine la AN como un episodio externalizado y no como una característica estructural del paciente. Vale decir, se dice que el o la paciente se encuentra con anorexia y no que es "anoréxico". Rechaza un enfoque crítico y culpógeno hacia la familia para reemplazarlo por responsabilidades de cuidado. Tiene como diferencia con el modelo original de Minuchin en que no se abordan aspectos de la interacción familiar hasta que no se haya mejorado y alcanzado un peso adecuado.

Utilizando este método se han publicado numerosos estudios que muestran su efectividad en distintos ámbitos, cuando se lo compara con grupos controles y con terapia individual de corte psicodinámico ${ }^{8}$.Todos los estudios publicados son de pequeño tamaño, con limitado poder para detectar diferencias clínicamente importantes, con diferencias en la selección de participantes y formas de reportar los resultados ${ }^{9}$. Casi siempre se reporta el peso al final del tratamiento, las recaídas y cuestionarios que evalúan el estado de salud de los pacientes con AN. Un punto crítico en estos estudios son los grupos controles en los que no se puede descartar contaminación y donde e seguimiento es limitado con respecto al grupo intervención. Es probable que esto ultimo pueda deberse a características específicas de esta patología, en la que resulta difícil mantenerse totalmente al margen de un tratamiento psicoterapéutico; por este motivo abundan estudios con diseños cuasi-experimentales.

Existen variaciones dentro de estos modelos, por ejemplo un estudio publicado recientemente evaluó una intervención apropiada para aquellos pacientes gravemente emaciados o bien para quienes presentan un rechazo alimentario persistente y mayor perturbación psicológica ${ }^{6}$ :la "sesión de comida". La "sesión de comida" realizada en este estudio incluía un trabajo motivacional previo con los pacientes, durante el cual se acordaba con ellos y con sus padres qué se iba a comer y beber durante dicha sesión y donde quedaban planteadas las decisiones tomadas como un desafío y un obstáculo por conquistar por parte de los involucrados. El objetivo era que los padres lograran que el paciente comiera en el consultorio y durante un período definido "un bocado más" que lo que habitualmente le permitía la enfermedad. La comida era traída para el paciente y los padres eran invitados a colaborar sólo si el paciente no lograba el cometido. Las características principales del método Maudsley se resumen en el cuadro 3 y sus fases en el cuadro 4. 
Cuadro 3: características principales del método Maudsley33.

- No asume que antes del tratamiento la familia fuese disfuncional.

- Se asume que no son los pacientes quienes generan las conductas de restricción y purga, sino la enfermedad.

- Los padres retoman en forma temprana el control de la alimentación y la restauración del peso adecuado.

- Los cambios estructurales de la familia son fundamentales, pero la restauración del peso y la seguridad física del paciente son el principal foco inicial.

- La duración habitual es de 25 sesiones desarrollada durante 12 meses.

Cuadro 4: fases de la terapia familiar con el método Maudsley.

\section{Realimentación}

Por medio de un trabajo de psicoeducación y con el acento puesto en la responsabilidad, en forma supervisada la familia procede a mejorar la alimentación del o la paciente hasta alcanzar el $90 \%$ del peso ideal.

\section{Trabajo transicional de control del adolescente}

Los adolescentes gradualmente asumen control de la alimentación con menor supervisón de los padres. Esta fase fina liza con la aparición de las menstruaciones o alcanzar el 100\% del peso ideal.

\section{Trabajo sobre temas de desarrollo del adolescente}

Se asiste a la familia para retronar al ciclo vital normal detenido por el episodio de AN y sobre nuevo patrón de interacciones familiares. Se consideran otros aspectos como problemas familiares no relacionados con AN, problemas de pareja, comorbilidades etc.

\section{Rehabilitación y consejo nutriciona}

Para aquellos pacientes que se encuentran por debajo de su peso ideal debe iniciarse un programa de rehabilitación nutricional ${ }^{10}$. Se sugiere una ganancia semanal de peso de 1 a $1,5 \mathrm{~kg}$ para los pacientes que estén internados; y de hasta $0,5 \mathrm{~kg}$ por semana para los ambulatorios. Usualmente, este objetivo se logra comenzando con 30 a $40 \mathrm{Kcal} / \mathrm{kg}$ y se las va aumentando progresivamente. En algunos pacientes se hace necesario indicar suplementos minerales y vitamínicos ${ }^{2}$. Cada vez que sea posible, la realimentación debe efectuarse por medios naturales y recurriendo a la comida habitual. En caso de que persistan los vómitos provocados o se agrave la pérdida de peso, se requiere una vigilancia de los pacientes durante al menos las dos horas posteriores a la ingesta. La desnutrición extrema o el rechazo total a alimentarse pueden conducir a la necesidad de una alimentación asistida.

En el proceso de realimentación existen ciertos parámetros que deben ser exhaustivamente monitoreados: los signos vitales, el ingreso y el egreso de fluidos, el equilibrio del medio interno; y la evaluación de la presencia de edemas y síntomas gastrointestinales, particularmente constipación y distensión².

La re-nutrición provoca un desplazamiento de agua hacia al sector exhacelular y plasmático (con eliminación de los edemas) y un aumento de la diuresis que puede ocasionar descenso de peso ${ }^{10}$.

El denominado "síndrome de realimentación" puede observarse en pacientes con anorexia nerviosa moderada a grave (un índice de masa corporal un 10\% menor al adecuado). Se desencadena entre la segunda y la tercera semana de la realimentación. Pueden aparecer trastornos cardíacos severos y delirios. Las causas estarían asociadas a disbalances electrolíticos sobre todo hipofosfatemia. Si bien no hay mucha bibliografía que lo sustente, muchos expertos coinciden en recomendar con función renal normal la administración de fósforo durante las primeras fases de realimentación ${ }^{3}$.

\section{Farmacoterapia}

Lo difícil del abordaje terapéutico de la anorexia nerviosa, sobre todo por lo poco alentadores que resultan los tratamientos en el largo plazo, ha llevado a evaluar el uso de varios psicofármacos. Sin embargo, todavía los resultados no son auspi$\operatorname{ciosos}^{10}$.

Los psicofármacos se limitan a prescripciones prudentes tanto para los trastornos de alimentación como para los estados comórbidos $^{11}$. Pueden pensarse como forma complementaria los ansiolíticos y los antidepresivos. Sin embargo, deben hacerse las siguientes aclaraciones. Los ansiolíticos implican riesgo de desarrollo de dependencia y también pueden ser usados en los intentos suicidas. De los antidepresivos, los inhibidores selectivos de la recaptación de serotonina (ISRS) reducen el apetito y el peso corporal, y provocan náuseas, diarrea y alteraciones del ritmo del sueño. El bupropion estaría contraindicado por inducción a las convulsiones. Un estudio con 35 pacientes con anorexia nerviosa de tipo restrictivo evaluó en forma exitosa el uso de fluoxetina en dosis de 20 a $60 \mathrm{mg} /$ día para el mantenimiento del peso corporal en una etapa de mantenimiento. En cuanto a los antidepresivos tricíclicos, se asocian a mayor sedación, efectos neurovegetativos y anticolinérgicos; generan mayor aumento del peso corporal, implican efectos cardiológicos y son más peligrosos en las sobredosis ${ }^{234}$. En el caso de que aparezca humor depresivo durante la internación, se sugiere no apresurarse con la medicación a menos que se trate de un episodio depresivo grave. Para los pacientes esta fase puede ser un momento de elaboración con expresión de emociones, dudas e incluso desesperación.

Respecto de la posible indicación de antidepresivos podemos definir cinco escenarios respecto de la relación entre los trastornos de alimentación y los cuadros depresivos.

a) Desarrollo de los síntomas afectivos consecutivamente a la aparición de los síntomas de bulimia o anorexia nerviosa, con mejoría de los mismos cuando mejora el estado de ánimo. Esto hace pensar en un síndrome depresivo secundario al trastorno alimentario.

b) Episodio depresivo mayor que aparece a partir de la mejoría del cuadro alimentario con resolución espontánea del trastorno del humor.

c) Desarrollo del cuadro alimentario y en etapas tempranas también del trastorno del humor; mejora el cuadro alimentario pero no se evidencia mejoría en la depresión.

d) El episodio depresivo aparece tiempo después de logrado el control de los síntomas del trastorno de la alimentación.

e) La depresión mayor precede el comienzo de los síntomas que configuran el trastorno alimentario.

Siguiendo este esquema la indicación de los antidepresivos estaría reservada ${ }^{7}$ para los tres últimos escenarios (c, d y e).

\section{Tratamientos específicos para los pacientes con bulimia}

Junto con otras modalidades de tratamiento, la orientación para los cuidados alimentarios puede ser útil para reducir los comportamientos relacionados con el desorden en el comer. Sería efectivo indicar reducir al mínimo la restricción alimentaria, aumentar la variedad de las comidas y alentar el ejercicio saludable pero no excesivo ${ }^{11}$. 


\section{Psicoterapia}

En pacientes con bulimia se ha desarrollado la terapia cognitivo comportamental para la bulimia nerviosa desarrollada específicamente para este trastorno. Su efectividad ha sido probada en numerosos ensayos clínicos controlados, por lo cual la guía de práctica del Reino Unido la considera indicada en la primera fase de tratamiento, considerándola evidencia grado A que parte de ensayos clínicos aleatorizados y controlados de buen calidad y consistencia .

Se desarrolla en 16 a 20 sesiones durante cuatro a seis meses. Revisiones sistemáticas muestran en forma consistente que este tipo de terapia aumenta los períodos de abstinencia, disminuye la frecuencia de atracones y mejora la depresión ${ }^{12}$.

Se trata de una terapia "manualizada" o semiestructurada y enfoca el tratamiento sobre los factores y los procesos que mantienen y perpetúan el problema, por sobre los que actuaron en el desarrollo inicial. El objetivo no sólo es un cambio en la conducta alimentaria si no una modificación en las ideas, los razonamientos y las percepciones que llevan a ella, lo que se denomina "reestructuración cognitiva", aprendiéndose además a identificar las situaciones gatillo que precipitan los atracones. Durante la primeras sesiones se pone énfasis en la mirada cognitiva para luego incluirse estrategias de educación alimentaria que mejoren el circuito que oscila desde una dieta restrictiva que genera hambre, hasta el atracón o utilización de conductas compensatorias de distintos tipos ${ }^{13}$.

Se reservan otros tipos de terapia de corte psicodinámico para aquellos pacientes que la rechazan o no me mejoran luego del tratamiento. Estas estrategias tiene similar efectividad a la anterior pero demandan el doble de tiempo, evidencia grado $\mathrm{B}^{714}$. Por otro lado, las técnicas psicodinámicas y psicoanalíticas indivuaduales o grupales pueden ser útiles una vez que los atracones y las purgas han mejorado o en pacientes que no los presentan habitualmente.

\section{Farmacoterapia}

A diferencia de la anorexia nerviosa, actualmente se manejan recursos terapéuticos farmacológicos genuinos, de cierta eficacia comprobada. De esta forma, el uso de antidepresivos tiene la finalidad de remitir o reducir la frecuencia de episodios de ingesta-purga. Es decir que motivarían su prescripción estos síntomas, para los cuales hay varios fármacos que han demostrado eficacia, independientemente del estado de ánimo del paciente. De todas formas, la tasa de pacientes sin respuesta sigue siendo elevada y las remisiones totales, escasas $^{15}$. Estas aclaraciones son válidas para no incrementar las dosis en forma innecesaria ni incurrir en la polifarmacia. De este modo, también se debe tener en cuenta las latencias de estas drogas que oscilan entre 20 y 40 días, aunque pueden observarse mejoras más precoces.

Al presente, todas las drogas de este grupo mostraron similar eficacia, por lo tanto su elección está en relación con el perfil de la droga (farmacocinética, toxicidad, efectos adversos) y con las características propias del paciente (ej. comorbilidades). De esa forma, entre los agentes más nuevos, el bupropion queda contraindicado por la mayor incidencia de convulsiones, sugiriéndose evitar aquellos que pueden determinar un aumento ponderal como la mirtazapina. Las dosis suelen ser similares que para la depresión mayor, a excepción de la fluoxetina que se usaría en dosis más elevadas. La duración de los tratamientos sería de al menos seis meses a un año a partir de la remisión ${ }^{1}$

Evidencia reciente muestra que la combinación de las intervenciones psicoterapéuticas y la medicación resultaría en probabilidades más altas de remisión. De esta forma algunos autores promueven la indicación farmacológica al inicio del tratamiento ${ }^{11}$. Otros, en cambio, son más cautos y reservan el uso de la farmacoterapia para después de haber obtenido algunos cambios a partir de abordajes no farmacológicos ${ }^{1}$.

Recibido el 30/05/07 y aceptado el 22/06/07

\section{Bibliografía}

1.Yager JDM: Guideline Watch Practice guideline for the treatment of patients with eating disorders, 2nd edition. Arlington, VA, American Psychiatric Association, 2005 2.Sadock BJ, Sadock VA, Kaplan HI: Kaplan \& Sadock's comprehensive textbook of psychiatry / editors, Benjamin J. Sadock, Virginia A. Sadock. Philadelphia, Lippincott Williams \& Wilkins, 2004

3. Lenoir M, Silber T. Anorexia nerviosa en niños y adolescentes Parte 2. Anorexia nerviosa Arch Argent Pediatr 2006;104(4):338-344

4. Corcos M AG, Bochereau D, Chambry et Jeammet PH.: Troubles des conduites alimentaires á ládolescence., in Encyclopédie Médico-Chirurgicale, vol 37-215-B-65. Paris, Elsevier, 2002, p 16p

5. Rauch Hercovici, Bay L. Anorexia nerviosa y bulimia: Amenazas a la autonomía. Terapia Familiar. Argentina: Paidos 1991:21-136

6. Herscovici C: La sesión de comida, el recupero de peso y su interacción con la psicopatología de la anorexia nerviosa adolescente. Vertex. Rev. Arg. de Psiaquiat. 2006; XVII (65):7-15

7. National Collaborating Centre for Mental Health: Eating disorders: Core interventions in the treatment and management of Anorexia Nervosa, Bulimia Nervosa and related eating disorders. London, National Institute for Clinical Excellence. January 2004

8. Le Grange D, The Maudsley family based treatment for adolescent anorexia nervosa. World Psychiatry 2006 4:3

9. Le Grande D, Lock J The dearth of psychological treatment studies for anorexia nervosa.. Int J Eat Disord 2005 Mar; 37: 79-9

10. Walsh BT, Klein DA: Eating disorders. Int Rev Psychiatry 2003; 15(3):205-16

11. Forman SM (ed): Traetment of anorexia nervosa and bulimia nevosa. 2003

12 Hay PJ, Baccatchut J. Psychotherapy for bulimia nervosa and binging. Cochrane Library Review 2006 issue 2 Chichester UK

13. Wikinski SJ, Gabriela Silvia: El tratamiento farmacológico en psiquiatría. Indicaciones, esquemas terapéuticos y elementos para su aplicación racional. Buenos Aires, Editorial médica panamericana, 2005

14Agras WS, Walsh BT, Fairburn CG A multicenter comparison of cognitive behavioural therapy and interpersonal therapy. for bulimia nervosa. Arch Gen Psychiatry 2000;54:459-65

15. Fernandez Rava M, Silber T. Bulimia Nerviosa, desde la etiología hasta la prevención. Arch Argent Pediatr 2004;102(6):468-474. 